\title{
Development of Hospital Formulary as a Patient Safety Drug Delivery System in Promoting Rational use of Drugs in Tertiary Care Hospital
}

\author{
Fatima Tahniyath ${ }^{* 1}$, Fatima Hafeez ${ }^{1}$, Hasbeen Sultana ${ }^{1}$, Mohammed Ashfaq Hussain ${ }^{1}$ and Syed Abdul \\ Azeez Basha'
}

${ }^{1}$ Pharm.D (PB), Department of Pharmacy Practice, Deccan School of Pharmacy, Hyderabad, Telangana (India) 2DM.Pharm, PhD, PGDM, PCCRM, Principal, Deccan School of Pharmacy, Hyderabad, Telangana (India).

\begin{abstract}
Objective: To constitute a Pharmacy Therapeutic Committee (PTC) and compile, design, develop and publish Owaisi Hospital and Research Centre (OHRC) Hospital Formulary for a tertiary care multi speciality hospital as a patient safety drug delivery system in promoting rational use of drugs. Methodology: PTC Committee was constituted and a descriptive form was designed for acquisition of data for drugs prescribed in respective departments and information pertaining to treatment modalities. The content of monographs to be incorporated was approved by PTC. The latest drug list was obtained from central pharmacy, respective departments/specialties and nursing stations. Subsequently, drugs were classified therapeutically along with USFDA pregnancy risk category; individual drug monographs were prepared, required ancillary information was added and lastly compared with WHO Essential Drugs List (EDL). Copies of the formulary were given to Medical Superintendent, Chief Medical Officer, and Chief Pharmacist and all the departments of Owaisi Group of Hospitals for implementation in the hospital as a tool to promote rational drug use. Results: PTC approved OHRC Hospital Formularyas a handy desk reference was compiled in 530 pages containing 306 generic drugs and FDCs. About 58 drugs were identified as Category A and B, 138 drugs as Category C, D, B/D, A/C and C/D and 7 drugs as Category X. Conclusion: Hospital formulary reflects the current clinical judgment of medical staff ensuring rational drug therapy, controlling drug costs, evidence based prescribing to reduce variation in the levels of treatment; additionally it helps in better inventory control by avoiding zero stock level and to limit the number of brands for each generic drug..
\end{abstract}

Key words: FDC-Fixed dose composition, Hospital Formulary, Inventory control, Monograph, Tertiary care, Rational drug use.

\section{INTRODUCTION}

Medicines play a crucial role in the prevention and treatment of diseases. When used correctly, they can offer simple and costeffective solutions to many health problems. A formulary system is the ongoing process through which a health care organization establishes policies regarding the use of drugs, therapies, and drug related products and identifies those that are most medically appropriate and cost-effective to best serve the health interests of a given patient population. ${ }^{1}$ Formulary systems are used in many different settings and organizations with policy statements on the use of formularies and formulary systems in hospitals and health care systems. ${ }^{2,3}$ Many products are minor variations of a prototype drug and offer no therapeutic advantage over other drugs that are already available, many new products for therapeutic indications are not relevant to the basic needs of the population. ${ }^{4}$ A number of other problems known to exist in most pharmaceutical system are limited drug budgets, increasing number of therapeutic alternatives, improper prescribing and use of medications, presence of unsafe and non-efficacious drugs, lack of unbiased drug information, high costs of handling large number of drugs, drugs of questionable quality on the market.,
Submitted date :06-Mar-2015 Accepted date :21-Mar-2015

DOI: $10.5530 / \mathrm{ijopp} .8 .1 .3$

Address for correspondence:

Miss. Fatima Tahniyath, Pharm D (PB) Internship student, H.No: 17-4-19, Outside Yakutpura, Hyderabad-500023, INDIA. Phoneno: 9948219786 E-mail:ftahniyath@gmail.com

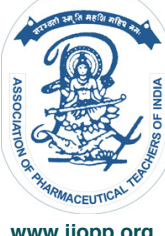

www.ijopp.org 


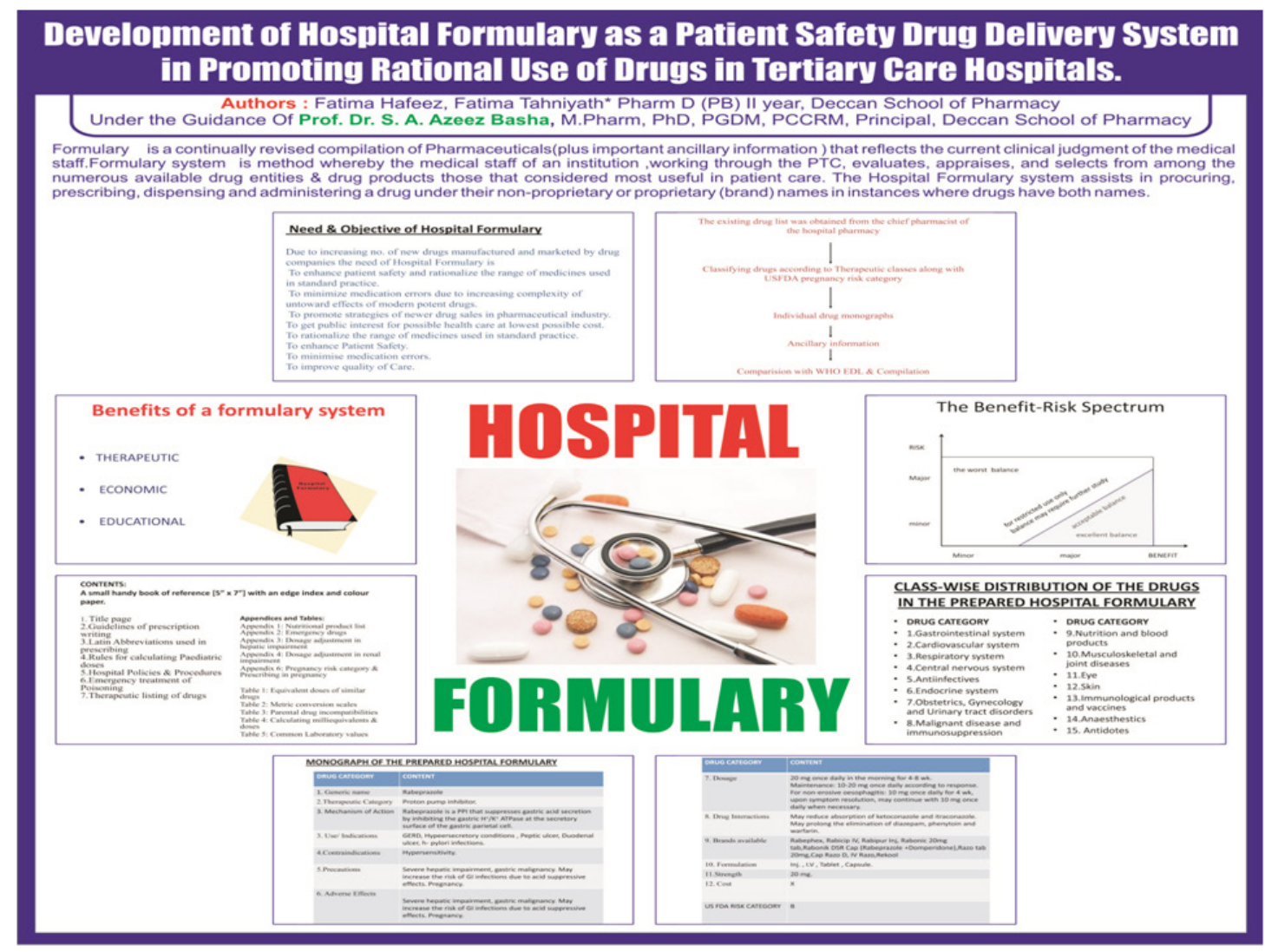

Graphical Abstract

Although National formulary exists in India, its use is limited due to non-availability of updated information. Hence, Hospital formulary system can be an asset for our health care system. ${ }^{7}$

A large number of drugs, drug variables and the multiplicity of drugs available for the treatment of diseases and making their use becomes complexity. Lack of co-ordination among the various specialties in treating patients is cited to be a major reason for medication errors and the lack of communication between health care providers contributes to more of adverse events.

In its efforts to promote safe and cost-effective use of medicines, World Health Organization (WHO) released the first edition of the WHO Model Formulary in 2002. This formulary is the first global publication to give comprehensive information on all 325 generic drugs contained in the WHO Model List of Essential Medicine. ${ }^{8}$ Correct use of this tool will improve patient safety and limit excess medical spending. Therefore, the WHO Model Formulary is primarily intended as a model, for national governments and institutions, as a basis for creating their own formularies. ${ }^{9}$ It has been stated that the main reason for developing a formulary is to promote rational prescribing. WHO also recommends in their policy perspectives on medicine that development of formulary system through Drugs and Therapeutic Committee in the hospitals will promote rational use of medicines. ${ }^{10}$

A hospital formulary provides information for the hospital staff about drug products approved for use by the Pharmacy and Therapeutic Committee . ${ }^{11,12}$

"The hospital formulary is a continually revised compilation of pharmaceuticals, which reflects the current clinical judgment of the medical staff including physicians, pharmacist and nurses and other experts in the diagnosis, prophylaxis, or treatment of disease and promotion of health". ${ }^{13}$

To enhance patient safety and minimize errors there is a need to develop a system mechanism so as to have clear and defined standards of care and protocols that are to be followed. A system therefore is needed to constitute a PTC/DTC (Pharmacy / Drug Therapeutic committee) that function with roles of advisory, educative and policy making body and to develop a Hospital formulary (HF) for the rational-safe, affordable and effective use of drugs and to reduce drug shortages and stock outs.

The study was conducted with the main objective of providing information on the available drugs in the hospital pharmacy to the physicians and the healthcare professionals by developing hospital's own formulary in 
comparison with WHO Model Formulary 2010, WHO and National list of Essential medicines 2010.

\section{MATERIALS AND METHODS}

\section{Study Period}

The study was conducted over a period of 6 months from November 2013 to May 2014.

\section{Study Site}

The study was conducted in a Tertiary Care multispecialty Hospital, Owaisi Hospital and Research Centre (OHRC), Hyderabad, Telangana.

\section{Hospital Background}

OHRC is a 1150 bedded multispecialty tertiary level referral and teaching hospital competently managed by Medical team of dedicated professionals, highly experienced in their respective specialities. Being a teaching hospital, there are no drug information sources present for the students. As the first step, our department started the drug information centre, ADR reporting and medication error monitoring and then worked on developing Hospital Formulary through PTC. By continuously updating the prepared formulary and conducting Drug Utilization Evaluation (DUE) programs, hospital formulary system can be made more effective.

\section{Study Design}

A prospective study was conducted to design, develop and publish hospital formulary for a tertiary care referral hospital with an aim to provide drug information to healthcare professionals for adopting standards of best practices for safe and effective drug therapy and better patient care.

\section{Pharmacy and Therapeutic Committee Approval}

A meeting was conducted with PTC and other health care professionals about the need and importance of Hospital Formulary. The contents of Hospital Formulary were approved by PTC. There were suggestions to add the general information, emergency drug list, immunological products and vaccines/sera and to incorporate methods/tables for dosage adjustments in renal and hepatic diseases and paediatric dosing.

The latest drug list of 1220 formulations including single and combination formulations was obtained from pharmacy, respective departments, specialties and nursing stations. Subsequently drugs were classified therapeutically along with USFDA pregnancy risk category. All drugs present in the drug list were systematically evaluated for their need, efficacy and safety. The Heads of all departments were asked to select the prefer- able brand/generic names of drugs or they can write drugs that have proven efficacy on their practice which are available/not available in hospital pharmacy. All their suggestions were considered. The doctors clearly informed that from their chosen drugs only cost effective, safe and quality drugs of at least 3 or 4 brands will be selected. The selected drugs were classified according to the British National Formulary as the standard format for the formulary list. Monographs were prepared for all the selected 379 drugs in the hospital pharmacy with the following contents: Synonyms, Therapeutic category, Indication, Pregnancy risk factor, Contraindications, Precautions/Warnings, Adverse reactions, Dosage, Drug interactions, Patient information, Brands available/strength/formulation.

Copies of the OHRC hospital formulary were given to all the health-care professionals, medical superintendent, chief medical officer, and chief pharmacist and to pharmacy and therapeutic committee who participated in the first survey.

The OHRC hospital formulary was compared with WHO model formulary 2010 on the following parameters.

- Number of drugs present.

- Class wise distribution of drugs.

- Number of brands available for generic drugs in Owaisi Hospital Formulary.

- Pregnancy category of drugs.

- Monograph content.

- Fixed drug combination.

\section{RESULTS}

\section{Class wise distribution of the drugs in the OHRC Hospital Formulary with WHO Model Formulary 2010}

\section{WHO Model Formulary 2010}

In the WHO model list the drugs under the gastrointestinal, cardiovascular, respiratory and central nervous systems were 12, 21, 6 and 24, respectively. Ninety two drugs were from anti-infective class. Drugs falling under endocrine system and obstetrics and gynaecology were 10 each. Musculoskeletal system comprised of 12 drugs and 31 preparations were used for eye, ear and skin disorders. Immunological and vaccines category includes 19 preparations. Fourteen were under the anaesthetic, 12 were under the category of antidotes as illustrated in Table 1.

\section{OHRC Hospital Formulary}

In the prepared hospital formulary, drugs under the gastrointestinal, cardiovascular, respiratory and central nervous systems were 24, 44, 17 and 61 respec- 
Table 1: Class wise distribution of the drugs in the OHRC Hospital Formulary with WHO Model Formulary 2010

\begin{tabular}{lcc}
\multicolumn{1}{c}{ Drug Category } & $\begin{array}{c}\text { Drugs as per } \\
\text { WHO }\end{array}$ & $\begin{array}{c}\text { Drugs in } \\
\text { OHRC,HF }\end{array}$ \\
\hline Gastrointestinal system & 12 & 24 \\
Cardiovascular system & 21 & 44 \\
Respiratory system & 6 & 17 \\
Central nervous system & 24 & 61 \\
Antiinfectives & 92 & 41 \\
Endocrine system & 10 & 25 \\
Obstetrics, Gynecology and & 10 & 6 \\
Urinary tract disorders & & \\
Nutrition and blood products & 22 & - \\
Musculoskeletal and joint & 12 & 25 \\
disease & & \\
Eye, Ear, Skin & 31 & 14 \\
Immunological Products and & 19 & 21 \\
Vaccines & & 10 \\
Anaesthetics & 14 & 4 \\
\hline Antidotes & 12 & \\
\hline
\end{tabular}

(Numbers mentioned indicates the drugs of particular therapeutic category present in WHO, National drug list and prepared hospital formulary) tively, while 41 were anti-infective agents. Endocrine system, obstetrics and gynaecology comprised of 25 and 6 drugs, respectively. Musculoskeletal system, eye disorders, Ear and skin disorders consisted of 25, 14 medicines, respectively. 10 drugs were anaesthetics, 4 antidotes respectively. 21 immunological and vaccines were included as shown in Table 1 and Figure 1.

\section{Monograph content}

The WHO Model Formulary contains Patient Information and cost which was not included in OHRC hospital formulary as according to Drug Price Control (DPCO) Order there is always a variance in the cost depending on the drug products or medicament used, the manufacturing company, the availability of product in the market, the treatment regimen of the patient etc. The cost of a drug varies hence, cost is not included in our hospital formulary though it is considered as an important parameter and supplementary information as cost effectiveness and cost benefit analysis methods are often used to prescribe a drug.

WHO Model Formulary monographs does not contain mechanism of action of the drugs which was included

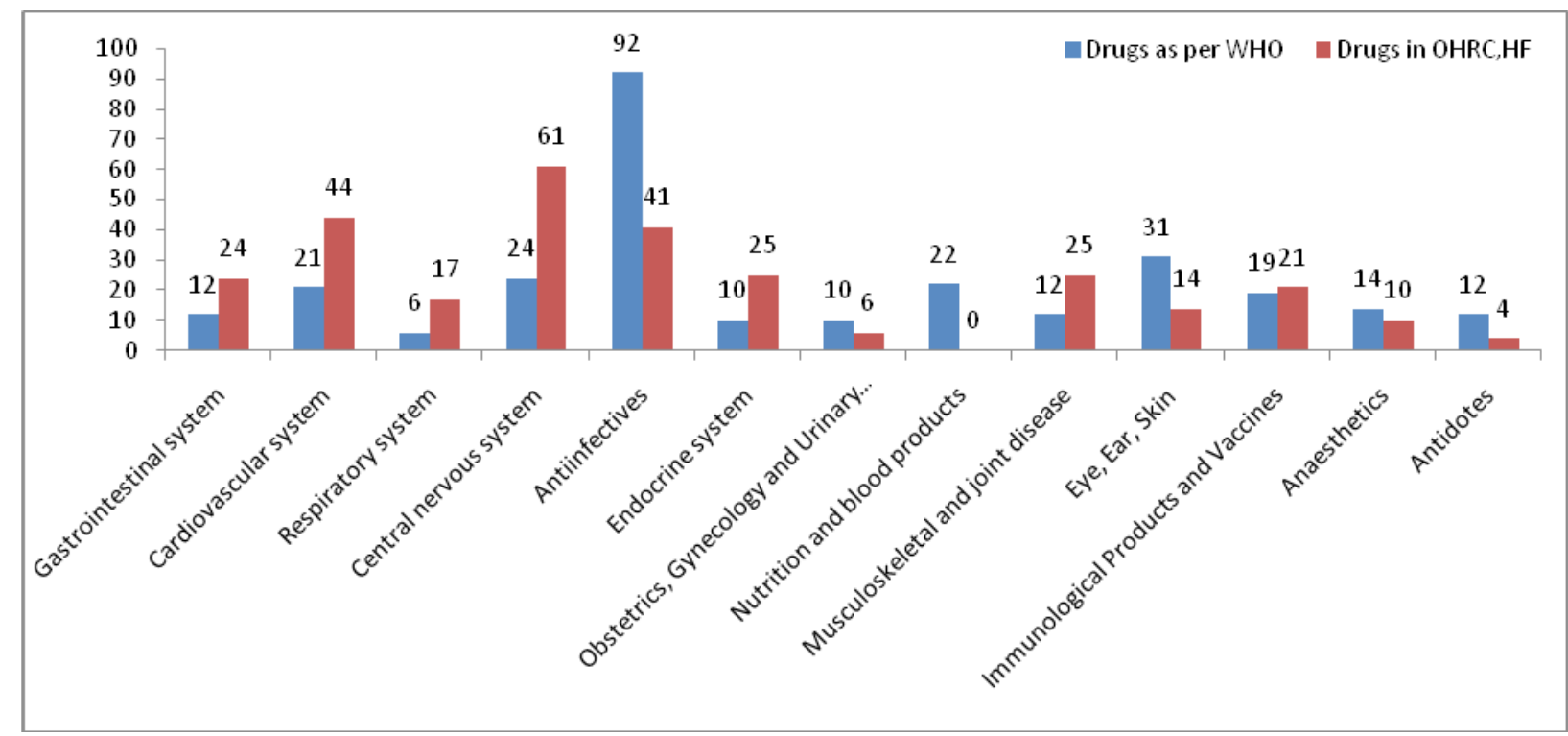

Figure 1: Shows class-wise distribution of the drugs in the OHRC Hospital Formulary with WHO Model Formulary 2010

in OHRC Hospital Formulary out of the authors' interest in order to provide complete information to the Health Care Team.

\section{Drugs to be avoided or used with caution in pregnancy}

Drugs should be prescribed in pregnancy only if the expected benefits to the mother are thought to be greater than the risk to the foetus. The WHO model formulary consists of 199 drugs and OHRC Hospital Formulary consists of 145 drugs, which should be avoided or used with caution in pregnancy. The number of drugs under each pregnancy category according to USFDA is shown in Table 3.

\section{DISCUSSION}

OHRC hospital formulary was compared with WHO Model Formulary 2010. In OHRC hospital formulary, each monograph consists of synonym, brand names, indications, contraindications, precautions, DI, ADR, dosage and strength shown in Table 2. Additionally, it 
Table 2: Comparison of monograph content of the OHRC Hospital Formulary with WHO Model Formulary 2010

\begin{tabular}{lcc}
\multicolumn{1}{c}{ Drug Category } & As per WHO & OHRC,HF \\
\hline Generic name & $\sqrt{ }$ & $\sqrt{ }$ \\
Therapeutic Category & $\sqrt{ }$ & $\sqrt{ }$ \\
Use/ Indications & $\sqrt{ }$ & $\sqrt{ }$ \\
Contraindications & $\sqrt{ }$ & $\sqrt{ }$ \\
Precautions & $\sqrt{ }$ & $\sqrt{ }$ \\
Adverse Effects & $\sqrt{ }$ & $\sqrt{ }$ \\
Dosage (Adult/ Paediatric) & $\sqrt{ }$ & $\sqrt{ }$ \\
Drug Interactions & $\sqrt{ }$ & $\sqrt{ }$ \\
Patient Information & $\sqrt{ }$ & X \\
Brands Available & $\sqrt{ }$ & $\sqrt{ }$ \\
Formulation & $\sqrt{ }$ & $\sqrt{ }$ \\
Strength & $\sqrt{ }$ & $\sqrt{ }$ \\
Cost & $\sqrt{ }$ & X \\
\hline
\end{tabular}

(Table represents presence or absence of particular category of information in WHO and prepared hospital formulary. $\sqrt{ }=$ Present,$x=$ Not present)

Table 3: Pregnancy Categories of the drugs present in the Hospital Formulary

\begin{tabular}{lc}
\hline USFDA Pregnancy Category & Number of Drugs \\
\hline A & 44 \\
B & 14 \\
C & 44 \\
D & 10 \\
X & 7 \\
A/C & 35 \\
B/D & 25 \\
C/D & 17 \\
\hline
\end{tabular}

USFDA- United States Food and Drug Administration.

also contains mechanism of action, pregnancy category, pharmacodynamic and pharmacokinetic parameters which are otherwise not included in WHO model formulary. Information on dosage adjustments in renal and hepatic diseases, paediatric dosing, lat in terms, equivalent doses of similar drugs and emergency drug list was added upon suggestion.

The most widely prescribed fixed dose combinations were analgesics, antimicrobials, multivitamins, and cold/ cough mixtures. While comparing the OHRC hospital formulary with WHO Model Formulary it was observed that the OHRC hospital formulary contains higher number of drugs in the following categories; Gastrointestinal System, Central Nervous System, Respiratory System, Cardiovascular System, Endocrine and Musculoskeletal and Joint Diseases. This correlates with the maximum utilization of the above mentioned category drugs in the different departments of the hospital. The following categories of drugs in the OHRC hospital formulary are less in number compared to WHO model formulary, i.e. eye, ear, skin, anti-infectives, anaesthesia and antidotes.

The number of drugs to be avoided or used with caution in pregnancy is less in OHRC hospital formulary compared to the WHO model formulary as only category C/D, D and X of the US FDA pregnancy category list and drugs whose pregnancy risk factor is not known have been included, whereas, WHO model formulary has given only the list of drugs and has not specified the pregnancy category. In the OHRC hospital formulary there are a total of 58 drugs identified as category $\mathrm{A}$ and $\mathrm{B}, 138$ drugs were identified as Category C, D, $\mathrm{B} / \mathrm{D}, \mathrm{A} / \mathrm{C}$ and $\mathrm{C} / \mathrm{D}$ and 7 drugs as Category $\mathrm{X}$ which are absolutely contraindicated in pregnancy.

While evaluating, it was found that the more number of drugs and drug combinations were present in the OHRC hospital formulary. The number of brands available for individual drug was higher than recommended.

\section{CONCLUSION}

The OHRC hospital formulary can be used as a vehicle to provide information to the physicians and healthcare professionals about the available drugs in the hospital pharmacy and can be used as a tool to rationalize the medicines used in the hospital. Implementation of hospital formulary helps in better inventory control by avoiding zero stock level.

Hospital formulary thus reflects the current clinical judgment of medical staff ${ }^{14,15}$ ensuring rational drug therapy, controlling drug costs and promotes high quality; evidence based prescribing thus reducing variation in the levels of treatment provided to patients ${ }^{16}$ and must be present in a complete, concise, updated and easy to use desk reference.

\section{CONFLICT OF INTEREST}

There are no conflicts to declare.

\section{ACKNOWLEDGEMENTS}

I sincerely acknowledge my deep sense of gratitude to Prof. Dr. Syed Abdul Azeez Basha, M.Pharm, Ph.D, PGDM, PCCRM, Principal, Deccan School of Pharmacy and Dr. MdIlyaz, B. Pharmacy, Pharm D (PB), H.O.D Department of Pharmacy Practice, Deccan School of Pharmacy, Hyderabad for their valuable guidance, constant encouragement, continued support and inspiration throughout the course of this work. 


\section{Highlights of Paper}

- Hospital Formulary system reduces medication errors, improves patient outcome.

- Formulary acts as a good inventory control measure.

- A vehicle by which medical and nursing staff promotes rational drug use.

- Handy desk reference for healthcare professionals.

\section{Author Profile}
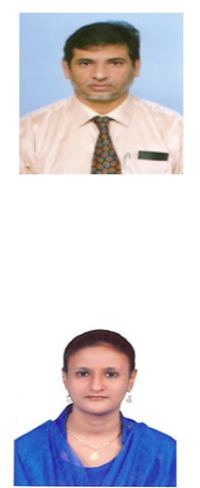

- Dr. Syed Abdul Azeez Basha: B.Pharm, M.Pharm, Ph.D, P.G. Dip. PMIR, PCCRM. He worked for 24 years as Teacher, Academician, Scientist and Administrator, served in Public and Private Organisations, Prominent Educational Institutions and as advisor and developer of Educational/Technical bodies in India and Abroad. Member of Indian Pharm Association (IPA), Indian Pharmacy graduate Association, (IPGA),Association of Pharmacy Teachers, India (APTI), Member of Indian Hospital Pharmacists Association (IHPA), Member of Libyan Pharmacy Council, Member of Saudi Pharmacy council. He served and executed Education regulation programs $(E R)$ through Pharmacy Council of India $(\mathrm{PCl})$ and has over 60 scientific publications to credit.

- Dr. Fatima Tahniyath: D.Pharm,B.Pharm, Pharm D (Post- Baccalaureate). Pharm D (PB) Internship Student of Deccan School of Pharmacy, Student member Association of Pharmacy Teachers, India (APTI). Was felicitated with Best Student of the year Award at B Pharm level.Participated in 5 National Conferencesand attended 2 workshops, gave 2 oral and 3 poster presentations and have 1 research article to credit

\section{REFERENCES}

1. Using medicines wisely by Farrah Khan: the place of the formulary in medicines management. Hospital pharmacist 2002; 9: 159- 63.

2. The pharmacy and therapeutic committee/ formulary system, Charles E. Daniels, quality assurance, chapter 59, 613-7.

3. ASHP technical assistance bulletin on drug formularies. drug formulary ASHP re-ports AJHP 1991; 48: 791-3.

4. Tyler, Sabrina W, Cole J. Russell May. ASHP Guidelines on the pharmacy and therapeutic committee and the formulary system, developed by an ASHP expert panel on formulary management: ed, Am J Health system pharm. 2008; 45: 1272-83.

5. Merchant SH, Qadry's, JS. A text book of hospital pharmacy. Hospital formulary; 2005-2006. 39-48.

6. Rajendran SD. Drug information. In G Parthasarathi, Hansen NK, Nahata MC. A textbook of clinical pharmacy practice. First publication. Orient Longman Private Limited. 2004; 267-78.

7. Subramanian G, Sreedhar D, Ranjith KA, et.al. Rationalization of drug therapy through national formulary of India: need of hours. Indian journal of hospital formulary. 2008; 45(3): 86-7.
8. WHO new Model Formulary-promoting consumer rights and patient safety. WHO Essential Drug Monitor 2003; 32: 10.

9. Furniss L. Formularies in primary care. Primary Care Pharmacy 2001; 1(1): 37-9

10. WHO policy perspective on medicines- promoting rational use of medicines core components; 2002.

11. Hoffmann PR. Perspectives on the hospital formulary. Hosp Pharm. 1984; 19(5): 359-61.

12. American Society of Hospital Pharmacy. ASHP statement on the pharmacy and therapeutics committee. Am J Hosp Pharm. 1986; 43: 2841-2.

13. William Hussan WE. The hospital formulary. In: Hospital pharmacy. $5^{\text {th }}$ ed. Philadelphia. 1986; 97-117.

14. Hassan WE. The Hospital formulary. In: Hospital Pharmacy. $5^{\text {th }}$ ed. Philadelphia: Lea and Febiger; 1986. p. 124-53.

15. ASHP technical assistance bulletin on hospital formularies. Am J Hosp Pharm. 1985; 42(1): 375-7.

16. Khan F. Using medicines wisely: The place of the formulary inmedicines management. Hosp Pharm. 2002; 9(1): 159-63. 